\title{
Reorganization of the nuclear lamina and cytoskeleton in adipogenesis
}

\author{
Valerie L. R. M. Verstraeten • Johan Renes • Frans C. S. Ramaekers • \\ Miriam Kamps • Helma J. Kuijpers • Fons Verheyen • Martin Wabitsch • \\ Peter M. Steijlen • Maurice A. M. van Steensel • Jos L. V. Broers
}

Accepted: 7 February 2011/Published online: 25 February 2011

(C) The Author(s) 2011. This article is published with open access at Springerlink.com

\begin{abstract}
A thorough understanding of fat cell biology is necessary to counter the epidemic of obesity. Although molecular pathways governing adipogenesis are well delineated, the structure of the nuclear lamina and nuclearcytoskeleton junction in this process are not. The identification of the 'linker of nucleus and cytoskeleton' (LINC) complex made us consider a role for the nuclear lamina in adipose conversion. We herein focused on the structure of the nuclear lamina and its coupling to the vimentin network, which forms a cage-like structure surrounding individual lipid droplets in mature adipocytes. Analysis of a mouse and human model system for fat cell differentiation showed fragmentation of the nuclear lamina and subsequent loss of lamins A, C, B1 and emerin at the nuclear rim, which coincides with reorganization of the nesprin-3/ plectin/vimentin complex into a network lining lipid droplets. Upon 18 days of fat cell differentiation, the
\end{abstract}

Electronic supplementary material The online version of this article (doi:10.1007/s00418-011-0792-4) contains supplementary material, which is available to authorized users.

V. L. R. M. Verstraeten $(\varangle) \cdot$ M. Kamps ·

P. M. Steijlen · M. A. M. van Steensel

Department of Dermatology, Maastricht University

Medical Centre, P. Debyelaan 25, PO Box 5800,

6202 AZ Maastricht, The Netherlands

e-mail: valerie.verstraeten@mumc.nl;

valerie.verstraeten@gmail.com

V. L. R. M. Verstraeten - F. C. S. Ramaekers - M. Kamps ·

P. M. Steijlen - M. A. M. van Steensel

GROW - School for Oncology and Developmental Biology,

Maastricht University Medical Centre, Maastricht,

The Netherlands

J. Renes

Department of Human Biology, Maastricht University

Medical Centre, Maastricht, The Netherlands fraction of adipocytes expressing lamins $\mathrm{A}, \mathrm{C}$ and $\mathrm{B} 1$ at the nuclear rim increased, though overall lamin $\mathrm{A} / \mathrm{C}$ protein levels were low. Lamin B2 remained at the nuclear rim throughout fat cell differentiation. Light and electron microscopy of a subcutaneous adipose tissue specimen showed striking indentations of the nucleus by lipid droplets, suggestive for an increased plasticity of the nucleus due to profound reorganization of the cellular infrastructure. This dynamic reorganization of the nuclear lamina in adipogenesis is an important finding that may open up new venues for research in and treatment of obesity and nuclear lamina-associated lipodystrophy.

Keywords LINC · Fat · Lamin · Adipose tissue . Nesprin-3 · Vimentin

\section{Introduction}

The incidence of obesity has increased dramatically in the past few decades, urging a thorough understanding of

J. Renes

NUTRIM - School for Nutrition, Toxicology and Metabolism, Maastricht University Medical Centre, Maastricht,

The Netherlands

F. C. S. Ramaekers - M. Kamps - H. J. Kuijpers - F. Verheyen ·

J. L. V. Broers

Department of Molecular Cell Biology, Maastricht University

Medical Centre, Maastricht, The Netherlands

F. C. S. Ramaekers - J. L. V. Broers

CARIM - School for Cardiovascular Diseases, Maastricht

University Medical Centre, Maastricht, The Netherlands

M. Wabitsch

Department of Pediatrics and Adolescent Medicine,

University Hospital of Ulm, Ulm, Germany 
adipocyte biology to counter this problem. Although the signaling pathways governing fat cell differentiation or adipogenesis are well-defined [reviewed in (Rosen and MacDougald, 2006)], the organization of nuclear infrastructure, i.e. the nuclear lamina and nuclear-cytoskeleton junction, in this process are not. The major building blocks of the nuclear lamina are lamins, nuclear intermediate filament proteins forming a fibrous meshwork lining the inner side of the nuclear envelope. Humans have three lamin genes: $L M N A$ encoding A-type lamins $\mathrm{A}, \mathrm{A} \Delta 10, \mathrm{C}$ and $\mathrm{C} 2$, $L M N B 1$ encoding lamin $\mathrm{B} 1$, and $L M N B 2$ encoding lamins $\mathrm{B} 2$ and B3. Lamins $\mathrm{C} 2$ and $\mathrm{B} 3$ are exclusively expressed in spermatocytes. Although the exact role of nuclear lamins is unknown, they are essential for maintenance of nuclear and cellular integrity, genomic integrity and gene expression [reviewed in (Broers et al. 2006; Verstraeten et al. 2007)]. Their role in cellular integrity is likely mediated through the proposed 'linker of nucleus and cytoskeleton' (LINC) complex (Crisp et al. 2006) comprising an interaction of lamins with the inner nuclear membrane (INM)-bound Sun proteins that associate with nuclear-cytoskeleton linker proteins in the outer nuclear membrane, i.e. nesprins. These proteins contain a binding domain for direct interaction with actin such as nesprin-1 and -2 , or in the case of nesprin-3 for plectin, an intermediate filament binding protein. Interestingly, mutations in $L M N A$ and $L M N B 2$ are associated with generalized and (acquired) partial lipodystrophy syndromes [reviewed in (Broers et al. 2006; Verstraeten et al. 2007)]. Therefore, we hypothesized that the nuclear lamina network, LINC and cytoskeleton are an integral part of adipogenesis and studied their structure in fat cell differentiation. We used two model systems, i.e. mouse 3T3-L1 and human preadipocytes derived from a patient with Simpson-Golabi-Behmel syndrome (SGBS) (Wabitsch et al. 2001).

\section{Materials and methods}

Cell culture and cellular Oil Red O accumulation

Mouse 3T3-L1 preadipocyte cell line was purchased from the American Type Culture Collection and cultured in DMEM-F12 (Cambrex, East Rutherford, USA) containing $10 \%$ fetal calf serum (FCS) and antibiotics in a 1:100 dilution (penicillin-streptomycin; GIBCO, Cat. No.15140148). Two days after reaching confluence, preadipocytes were induced to differentiate into adipocytes by culturing in DMEM-F12 containing 10\% FCS, 0.5 mM 3-isobutyl-1methyl-xanthine (Sigma, St. Louis, USA), $1 \mu \mathrm{M}$ dexamethasone (Sigma) and $5 \mu \mathrm{M}$ troglitazone (VWR, Amsterdam, the Netherlands) for 2 days, followed by 18 days in DMEM-F12 containing 10\% FCS and $1 \mu \mathrm{M}$ insulin (Sigma). A human preadipocyte cell line derived from a patient with SGBS was provided and characterized by Dr. Martin Wabitsch. SGBS cells were cultured and differentiated according to existing protocols (FischerPosovszky et al. 2008; Wabitsch et al. 2001). Differentiation was monitored by the visual appearance of fat droplets in the cells. At all time points, cells were fixed with $3.7 \%$ formaldehyde in DMEM-F12 for $10 \mathrm{~min}$ at room temperature (RT). The fixative was discarded and cells were washed with $\mathrm{H}_{2} \mathrm{O}$ followed by an additional wash with $70 \%$ ethanol and incubation with a filtered Oil Red O (ORO, Merck, Darmstadt, Germany) solution (1\% in isopropanol) for $30 \mathrm{~min}$ at RT. Images of the cells were taken with a Leica phase contrast microscope equipped with digital image acquisition.

Immunocytochemical analysis of cultured

3T3-L1 and SGBS cells

3T3-L1 and SGBS cells were cultured and grown on glass coverslips in 12-well culture plates. Before induction of differentiation and 3, 10, 18 days after induction of 3T3-L1 cells and, 5 and 10 days after induction of SGBS cells, cells were fixed with $4 \%$ formaldehyde in phosphate buffered saline (PBS) pH 7.4 for $15 \mathrm{~min}$, followed by permeabilization in $0.1 \%$ Triton $\mathrm{X}-100$ for $15 \mathrm{~min}$ at RT. Cells stained with the $\beta$-actin and BV-1118 antibodies were fixed in methanol at $-20^{\circ} \mathrm{C}$ for $10 \mathrm{~min}$. Primary antibodies (view list below) diluted in PBS containing 3\% bovin serum albumin (BSA) were applied onto the cells for $1 \mathrm{~h}$ at RT. After extensive washing in PBS, secondary antibodies were applied for $1 \mathrm{~h}$ at RT. Secondary antibodies used are fluorescein isothiocyanate (FITC)-conjugated rabbit anti-mouse Ig (1:100, DAKO, Glostrup, Denmark), FITC-conjugated swine anti-rabbit Ig (1:80, DAKO), Texas-Red-conjugated goat anti-mouse Ig (1:80, ITK-SouthernBiotech, Uithoorn, the Netherlands). Secondary antibodies were also diluted in PBS with 3\% BSA. After three final washing steps (each $5 \mathrm{~min}$ ) in PBS, slides were mounted in $90 \%$ glycerol, $0.02 \mathrm{M}$ Tris- $\mathrm{HCl} \mathrm{pH} \mathrm{8.0,}$ $0.8 \% \mathrm{NaN}_{3}$ and 2\% 1, 4-di-azobicyclo-(2,2,2)-octane (DABCO; Merck) containing $0.5 \mu \mathrm{g} / \mathrm{ml}$ propidium iodide (PI) or $0.5 \mu \mathrm{g} / \mathrm{ml}$ 4'-6-diamidino-2-phenylindole (DAPI) in case of double stainings. Hereafter, 3T3-L1 and SGBS cells were analyzed by confocal laser scanning microscopy. The applied settings of the Bio-Rad MRC600 confocal microscope (Bio-Rad Laboratories Ltd, Hemel Hempstead, UK) have been previously described (Verstraeten et al. 2006).

Each fat cell differentiation experiment of 3T3-L1 and SGBS cells was performed at least three independent times, and in each experiment over 300 cells were imaged/evaluated for a specific protein labeling at the aforementioned time points in differentiation. For quantification purposes, 
two independent investigators studied the nuclear lamina organization of lamins $\mathrm{A}, \mathrm{C}, \mathrm{B} 1$ and emerin in 3T3-L1 preadipocytes (i.e. cells that lack any lipid accumulation) and adipocytes before and 3,10 and 18 days following induction of differentiation. The labeling of the nuclear rim was scored as strong/moderate/weak-to-absent or fragmented. The nuclear lamina of adipocytes could be scored upon 10 and 18 days of differentiation. To compare fragmentation versus non-fragmentation or lack versus presence of nuclear rim expression (a binomial event) between different conditions, results were analyzed by a Fischer's exact test. Data were expressed as the sample proportion \pm S.E. A two-tailed value $P \leq 0.05$ was considered significant.

Primary antibodies used for immunofluorescence studies

Detailed description of the antibodies 133A2 (to lamin A and $\mathrm{A} \Delta 10$ ), RalC (to lamin C), Lamin B1, NCL-Emerin and $\beta$-actin (dilution for immunocytochemistry: $1 / 500$ ) has been provided in (Verstraeten et al. 2006).

\section{Additional antibodies}

- X223 (mouse IgG1) directed against lamin B2 (Hoger et al. 1990), kindly provided by Dr. G. Krohne (Würzburg, Germany). Dilution used for immunocytochemistry: $1 / 5$.

- Nesprin-3 is an affinity-purified rabbit polyclonal antibody to nesprin-3, raised against the seventh spectrin repeat common to both nesprin- $3 \alpha$ and $-3 \beta$, kindly provided by Dr A. Sonnenberg (Netherlands Cancer Institute, Amsterdam, the Netherlands) (Wilhelmsen et al. 2005). Dilution used for immunocytochemistry: $1 / 500$.

- HD-121 is a mouse monoclonal antibody against plectin/HD1. It was kindly provided by W.H. Irwin McLean (University of Dundee, Dundee, UK) and Katsushi Owaribe (University of Nagoya, Nagoya, Japan) (Hieda et al. 1992). Dilution used for immunocytochemistry: 1/100.

- P62 (mouse IgG2b) (Transduction Laboratories, Lexington, KY, USA) directed to nucleoporin p62 (Carmo-Fonseca et al. 1991). Dilution used for immunocytochemistry: 1/300.

- E7 (mouse IgG1) (Developmental Studies Hybridoma Bank, Iowa City, USA) against $\beta$-tubulin. Dilution used for immunocytochemistry: $1 / 25$.

- BV-1118 (mouse IgM) kindly supplied by Dr C. Viebahn (Bonn, Germany) directed against vimentin. Dilution used for immunocytochemistry: 1/10.
- Texas Red-X phalloidin (Molecular Probes, Eugene, OR 97402 USA) binding F-actin. Dilution used for actin labeling: $1 / 100$.

- GTU-88 (mouse IgG1) (Sigma-Aldrich) against $\gamma$-tubulin for staining of the microtubule-organizing center (MTOC). Dilution used for immunocytochemistry: $1 / 3000$.

- IID8 (mouse IgG1) (Affinity BioReagents, Golden, Colorado, USA) recognizing the sarco/endoplasmic reticulum (ER) $\mathrm{Ca}^{2+}$-ATPase SERCA2. Dilution for immunocytochemistry: 1/100.

2D gel electrophoresis, mass spectrometry and western blot analysis

Mouse 3T3-L1 preadipocytes were kept in DMEM-F12 containing $10 \%$ FCS and penicillin-streptomycin antibiotics in a 1:100 dilution. Two days after reaching confluence, preadipocytes were induced to differentiate into adipocytes by culturing in DMEM-F12 containing $10 \%$ FCS, $0.5 \mathrm{mM}$ 3-isobutyl-1-methyl-xanthine, $1 \mu \mathrm{M}$ dexamethasone and $10 \mu \mathrm{M}$ prostaglandin I2 (Biomol, Plymouth Meeting, PA, USA) for 1 day, followed by 18 days in DMEM-F12 containing $10 \% \quad$ FCS and $1 \mu \mathrm{M}$ insulin (Sigma). Whole cell protein lysates were obtained from mouse 3T3-L1 cells before and 18 days following fat cell differentiation as previously described (Renes et al. 2005). The protein samples were processed for $2 \mathrm{D}$ protein gel electrophoresis and differentially expressed protein spots were subsequently analyzed by mass spectrometry as previously described (Bouwman et al. 2004). Western blot analysis using the aforementioned primary antibodies RalC and $\beta$-actin was performed as previously described (Verstraeten et al. 2006).

Light and electron microscopy of a human adipose tissue specimen

For light and transmission electron microscopy, a skin biopsy specimen was fixed in 3\% glutaraldehyde buffered with $90 \mathrm{mM} \mathrm{KH} \mathrm{PO}_{4}(\mathrm{pH}$ 7.4). After fixation with $2 \% \mathrm{OsO}_{4}$ (Agar Scientific, Stansted, UK) in $0.1 \mathrm{M}$ veronalacetate buffer followed by impregnation in $1 \%$ veronalacetate ( $\mathrm{pH}$ 5.2) (Ladd Research Industries, Burlington, VT), the samples were dehydrated in graded series of ethanol and routinely embedded in Epon (Ladd Research Industries). $1 \mu \mathrm{m}$ thick sections were stained with toluidine blue for light microscopy. Ultrathin sections were counterstained with uranium acetate and lead citrate prior to examination in a Philips CM100 electron microscope. 


\section{Results}

Reorganization of the nuclear lamina and nuclearcytoskeleton coupling in fat cell differentiation

We used Oil-Red $\mathrm{O}$ staining of the intracellular fat content to monitor fat cell differentiation (Fig. 1a-d). 3T3-L1 cells were evaluated before and 3,10 and 18 days after induction of fat cell differentiation. SGBS cells were studied before, and 5 and 10 days following induction. Lipid accumulation occurred in small or large lipid droplets (Fig. 1c, d). Labeling of the intermediate filament protein vimetin showed previously identified well-defined cages around small lipid droplets (Fig. 1e, arrow) (Franke et al. 1987). Adipocytes with large droplets revealed a rather cortical organization of vimentin lining the plasma membrane (Fig. 1e, top cell). Careful analysis of the lamin A network in 3T3-L1 cells uncovered a dynamic reorganization of the lamina network upon induction of fat cell differentiation (Fig. 1f). Lamin A expression was observed within the nucleoplasm and particularly, at the nuclear periphery of preadipocytes that were primed to differentiate (Fig. 1f1). Hereupon, the lamin A network showed fragmentation (Fig. 1f2, 3), resulting in loss of lamin A expression at the nuclear periphery of up to $50 \%$ of adipocytes at 10 days of fat cell differentiation (Fig. 1f 4-6; $1 \mathrm{~g})$.

In 3T3-L1 and SGBS preadipocytes all lamin subtypes were expressed at the nuclear rim and in the nucleoplasm, whereas the integral INM-bound emerin was organized at the nuclear rim (Fig. 2a, panels 1, 5, 9, 13, 17 and Fig. 2d, panels $1,3,5,7,9)$. Nesprin-3 was mostly defined to the ER in $85 \%$ of 3T3-L1 preadipocytes (Figs. 2a21, 3a2), as concluded from its co-localization with SERCA2, an ER $\mathrm{Ca}^{2+}$-ATPase (Fig. 3c3). Plectin, which binds nesprin-3, partially co-localized with vimentin (Figs. 3d3, 3e3). Three days upon induction of differentiation, a stage at which lipid accumulation could not yet be detected, nucleoplasmic expression for lamins A, C and B1 was reduced in favor of a stronger nuclear lamina network that however, subsequently fragmented (Fig. 2a, panels 2, 6, 10, 18). Nuclear counterstaining with PI was normal in nuclei with a fragmented nuclear lamina network (data not shown). The lamin B2 network was not affected (Fig. 2a14). Up to $20 \%$ of preadipocytes primed to differentiate for 3-10 days showed fragmentation of the nuclear rim for lamin A, 10\% for lamin C and $\sim 2 \%$ for lamin B1 and emerin (Fig. 1h, Suppl. Table 1). Hereafter, emerin reorganized away from the nuclear rim to the ER (Fig. 2a19). Upon 10 days of fat cell differentiation, $\sim 50 \%$ of adipocytes eventually showed lack of lamin A (Fig. 2a4), 30\% lack of lamin C (Fig. 2a8), 50\% lack of lamin B1 (Fig. 2a12) and $\sim 70 \%$ lack of emerin (Fig. 2a20) at the nuclear rim (Fig. 1i,
Suppl. Table 1). Double labeling of 3T3-L1 cells showed initial loss of emerin, followed by that of lamin $\mathrm{A}$ and subsequently, of lamin C (Fig. 2b). Upon 18 days of differentiation, the fraction of adipocytes expressing lamins $\mathrm{A}, \mathrm{C}$ and $\mathrm{B} 1$ at the nuclear lamina increased significantly (Fig. 1i; Suppl. Table 1). However, 2D gel electrophoresis studies with subsequent mass spectrometry showed that the overall lamin A protein level at this time point of differentiation remained low (Fig. 2e). In previous 2D gels, we already demonstrated a reduced expression of the other Lmna gene product lamin C upon 18 days of fat cell differentiation (Renes et al. 2005), which we now confirmed by western blot analysis (Fig. 2f).

Nuclear pore complexes seem unaffected as evidenced by the organization of nucleoporin NUP62 at the nuclear rim of adipocytes (Fig. 2c2). Nesprin-3 moved away from the ER to the nuclear rim upon confluency (Fig. 2a21, insert) and, to the largest extent, upon induction of differentiation, with $85 \%$ of 3T3-L1 cells expressing nesprin-3 solely at the nuclear envelope (Figs. 2a22, 3a5). Consistent with its binding affinity for nesprin-3, plectin localized to the nuclear envelope upon induction of differentiation (Figs. 2d11 insert, 3d5 and 3e5), suggestive for recruitment of plectin to the nuclear rim by nesprin-3 as previously reported (Wilhelmsen et al. 2005). Hereafter, nesprin-3 reorganized away from the nuclear envelope to the ER surrounding lipid droplets in $85 \%$ of 3T3-L1 adipocytes (Figs. 2a23, 24 and 3a8). Co-localization studies with SERCA2 confirmed the redistribution of nesprin-3 to the ER (Fig. 3c6). Franke et al. (1987) previously reported a lipid droplet-associated ER network and described a cagelike meshwork of vimentin surrounding individual lipid droplets in 3T3-L1 adipocytes. We found that nesprin-3 and vimentin formed an intricate network around each lipid droplet, without co-localization (Fig. 3b, panels 12 and 18). As expected, plectin, which connects nesprin-3 to vimentin, co-localized with vimentin at the lipid droplets in 3T3-L1 adipocytes (Fig. 3d9). In SGBS cells, this co-localization was less obvious (Fig. 3e9) with more plectin remaining at the nuclear envelope of most adipocytes (Figs. 2d12, 3e8, f2).

Reorganization of the cytoskeleton in adipogenesis

Aside from vimentin, which reorganized from a filamentous network (Fig. 4a4, b3) to a network surrounding lipid droplets in adipocytes (Fig. 4a5, b4) and a cortical network in adipocytes with large lipid droplets (Fig. 4a6), actin and microtubules also reorganized profoundly. Induction of fat cell differentiation made actin organization change from mostly stress-fibers (Fig. 4a1, b1) to a cortical network surrounding wide, deep plasma membrane invaginations (Fig. 4a2), previously described as 'caves' (Parton et al. 

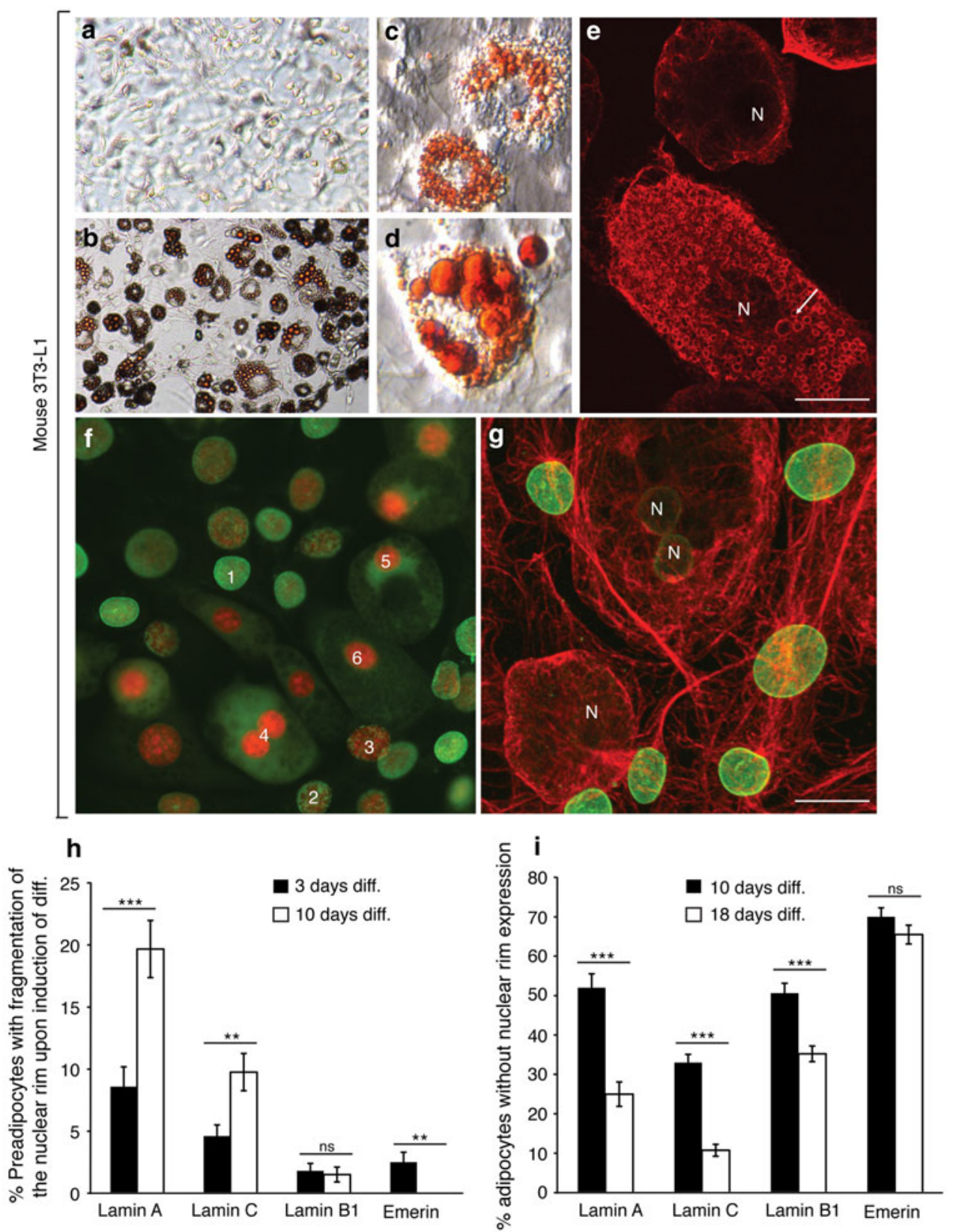

Fig. 1 Reorganization of the lamin A network during adipose conversion in 3T3-L1 cells. ORO staining of preadipocytes (a) and upon 9 days of differentiation (b-d) showed lipid accumulation in small (c) and large (d) lipid droplets. Vimentin formed a filamentous cage-like structure surrounding small lipid droplets (e, Z-projection of confocal stack; arrow indicates "cages") and reorganized to a predominantly cortical network in adipocytes with large lipid droplets (position of the nucleus is indicated by $\mathrm{N}$ ). Lamin A was gradually lost upon stimulation to fat cell differentiation [f1-6 green epifluorescence indicating lamin A; nuclear counterstaining using PI (red)]. 3T3-L1 preadipocytes showed a homogenous distribution of lamin A at the nuclear rim and in the nucleoplasm (f1). Shortly upon induction of differentiation the lamin A network became fragmented (f2, 3). Some lipid-containing adipocytes showed a cytoplasmic lamin A expression $(\mathbf{f 4}, \mathbf{5})$, and in about $50 \%$ of the adipocytes at 10 days of

differentiation, the nuclear rim was devoid of lamin A (f4-6). g Z-projection of a confocal image stack through adipocytes doublelabelled for lamin A (green) and microtubules (red) revealed a cortical organization of microtubules and loss of lamin A in mature adipocytes (the nuclei are indicated by $\mathrm{N}$ ). $\mathbf{h}$ Analysis of the nuclear lamina in 3T3-L1 preadipocytes that were primed to differentiate for 3-10 days showed up to $20 \%$ fragmentation of the nuclear rim for lamin A, $10 \%$ for lamin C and $\sim 2 \%$ for lamin B1 and emerin. i Mouse 3T3-L1 adipocytes showed a weak-to-absent nuclear rim expression of lamin A ( 50\%), lamin C ( $\sim 30 \%)$, lamin B1 ( 50\%) and emerin $(\sim 70 \%)$ upon 10 days of differentiation. After 18 days of fat cell differentiation, significantly more adipocytes expressed lamins A, C and B1 at the nuclear rim. More details provided in Suppl. Table 1. Scale bar indicates $10 \mu \mathrm{m}$. $* * P<0.005$; ***P $<0.001$ 

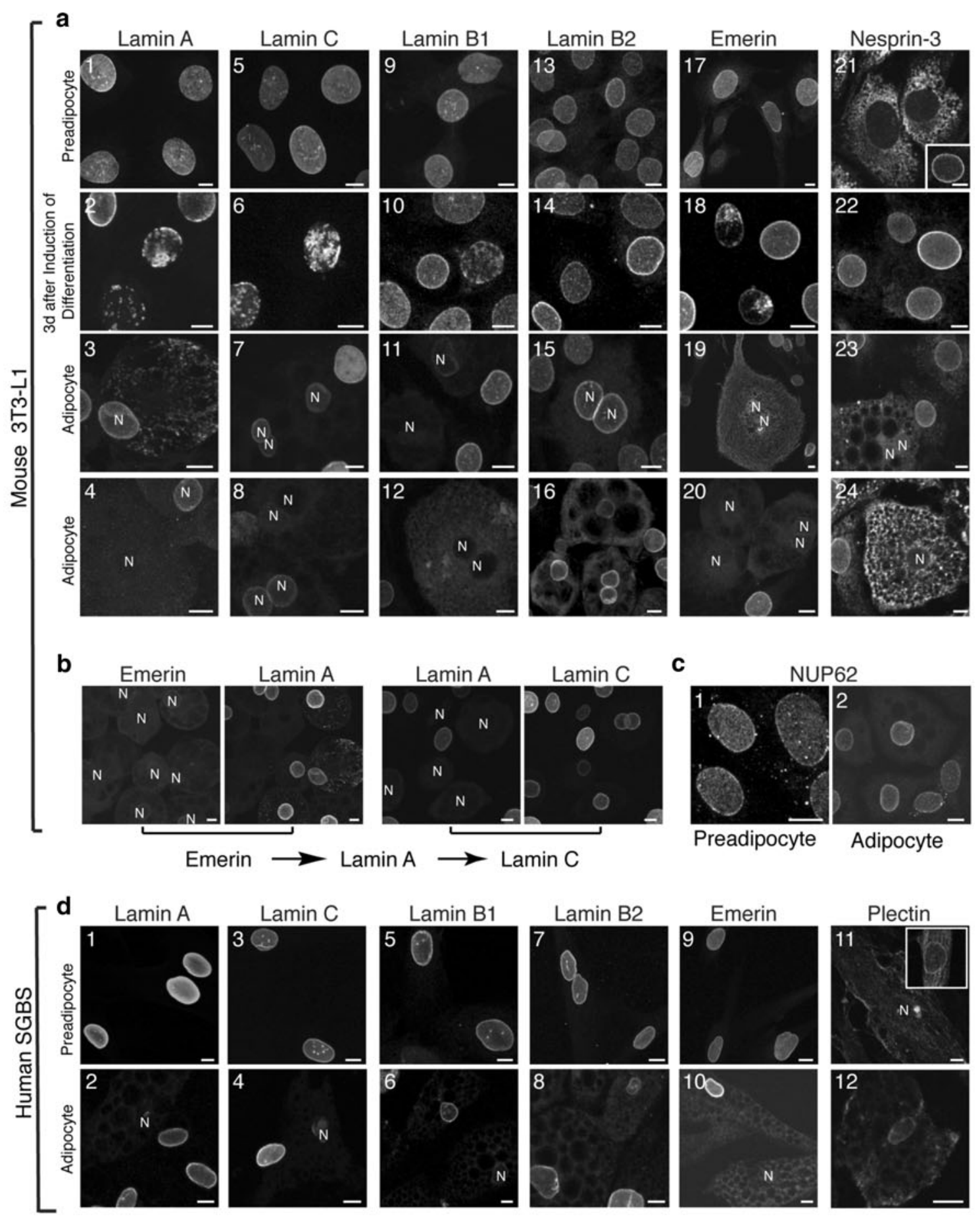

Lamin B1
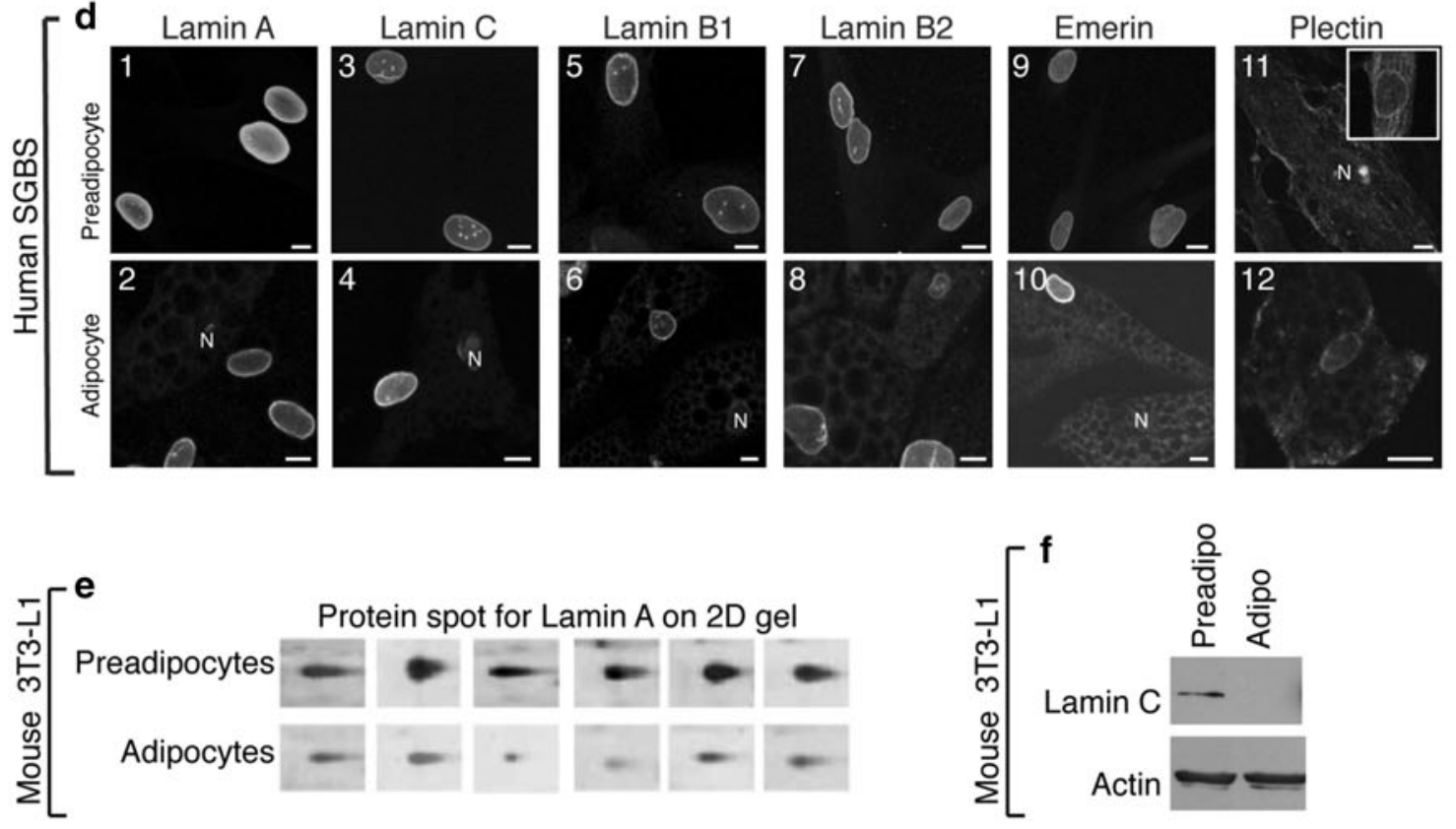
Fig. 2 Nuclear lamina breakdown in fat cell differentiation. Preadipocytes show a homogenous distribution for lamins and emerin at the nuclear envelope (a1, a5, a9, a13, a17; d1, d3, d5, d7, d9). Upon induction of differentiation, lamins $\mathrm{A}, \mathrm{C}$ and $\mathrm{B} 1$ accumulate at the nuclear rim, followed by lamina fragmentation (a2, a6, a10, a18), except for lamin B2 (a14). Emerin reorganizes away from the nuclear rim to the ER (a19). Nesprin-3 reorganizes from the ER (a21) to the nuclear rim upon confluency (a21, insert) and upon induction of differentiation (a22), followed by dissociation from the lamina to lipid-bound ER (a23, 24). From double-labeling studies we learned that emerin reorganizes first, followed by lamin $\mathrm{A}$, and then lamin $\mathrm{C}$ (b). Nucleoporin p62 remains at the rim throughout differentiation (c). Adipocytes show reduced expression of $(\mathbf{a 3}, 7, \mathbf{1 1})$ and eventually lack lamin A (a4, d2), C (a8, d4), B1 (a12, d6) and emerin (a20, d10). Plectin reorganizes from a more diffuse cytoskeletal distribution (d11) to the nuclear rim upon induction of differentiation (d11, insert) and redistributes into a network in between fat droplets in adipocytes (d12). e 2D protein gel electrophoresis and subsequent mass spectrometry of 3T3-L1 cells before treatment (preadipocytes) and 18 days upon induction of fat cell differentiation (adipocytes) showed a reduced expression of lamin A (protein spot with accession number p11516 in the Swissprot database) in adipocytes (six replicates derived from three independent experiments). f Western blot analysis confirmed reduced expression of lamin $\mathrm{C}$ in 3T3-L1 mouse adipocytes upon 18 days of fat cell differentiation. Bar, $10 \mu \mathrm{m} . N$ nucleus. All images result from $Z$-projection of the corresponding confocal image stack

2002), protruding into the perinuclear region. In adipocytes, the number of perinuclear caves decreased and a mostly cortical actin network remained (Fig. 4a3, b2). The microtubule network emanating from the centrosomes in preadipocytes (Fig. 4a7, b5), reorganized into a network in between lipid droplets (Fig. 4a8, b6) and under the plasma membrane (Fig. 4a9) in adipocytes. Interestingly, this profound reorganization of the nuclear lamina and cytoskeleton did not affect the distribution of the MTOC (Fig. 4a10, 11, b7, 8).

Structural analysis of adipocytes in a human adipose tissue specimen

Light (Fig. 5a1, 2) and electron (Fig. 5b1-4) microscopy of a subcutaneous adipose tissue specimen showed peripherally located thin ovoid-shaped nuclei that were often indented by lipid droplets (Fig. 5a2, b1, 3), as concluded from the lipid bi-layer of the nuclear envelope that surrounds indenting lipid droplets (arrow in Fig. 5b4). A filamentous network could be detected in between the nucleus and the lipid mass of the adipocyte (arrow in Fig. 5b2). Interestingly, no such filamentous network could be seen surrounding small lipid droplets indenting the nucleus (Fig. 5b4).

\section{Discussion}

Since the identification of the LINC complex (Crisp et al. 2006), researchers have tried to find more evidence for the nuclear-cytoskeleton coupling. The herein described dynamic organization of nuclear lamina structure, which coincides with profound changes in the cytoskeleton throughout fat cell differentiation further supports the importance of the LINC complex in physiology. Although our findings do not directly illustrate the necessity of nuclear lamina breakdown in reorganization of the cytoskeleton, other groups have shown that over-expression of wild-type or mutant lamin A disrupts fat cell differentiation and lipid accumulation, whereas complete lack of lamin A conversely promotes adipogenesis (Akter et al. 2009; Boguslavsky et al. 2006; Capanni et al. 2005). These reports underscore our results and hint towards the necessity of nuclear lamina reorganization in adipogenesis. Moreover, at 10 days of fat cell differentiation, a large fraction of adipocytes showed lack of lamins A ( 50\%), C $(\sim 30 \%)$, B1 $(\sim 50 \%)$ and emerin $(\sim 70 \%)$ at the nuclear rim. Interestingly, however, upon 18 days of differentiation, significantly more adipocytes expressed lamins A, C and $\mathrm{B} 1$ at the nuclear lamina. It is conceivable that upon continued differentiation the cells exhibit a different adhesion capacity and tend to detach more easily, particularly those adipocytes lacking the above nuclear envelope proteins. On the other hand, the loss of lamins A, C and B1 could be a temporary one needed for the initial steps of adipose conversion and cells might regain nuclear rim localization hereafter. Yet, protein analysis studies using samples obtained at 18 days of differentiation [Fig. 2e, $\mathrm{f}$ (Renes et al. 2005)] and studies performed by others (Tilgner et al. 2009) showed an evident decrease in protein levels of lamins $\mathrm{A} / \mathrm{C}$ and emerin upon adipose conversion.

Emerin is thought to be a key player in adipogenesis because of its role in nuclear-cytoplasmic shuttling of $\beta$-catenin (Markiewicz et al. 2006). Moreover, cells deficient in emerin have an increased adipogenic potential (Tilgner et al. 2009). Therefore, it is not surprising that our studies show an initial loss of emerin upon induction of adipogenesis, preceding the reorganization/loss of lamins $\mathrm{A}$ and $\mathrm{C}$ at the nuclear rim. Except for lamin B2, all lamin subtypes and emerin dissociated away from the nuclear envelope upon induction of differentiation. We therefore hypothesize that the absence of most lamin subtypes and particularly, that of lamins A and C, i.e. the major contributors to nuclear stiffness (Lammerding et al. 2006), results in enhanced plasticity of the nucleus. The latter may add to the multitude of nuclear indentations by lipid droplets detected in our human adipose tissue specimen.

Mutations in LMNA are associated with partial or generalized forms of lipodystrophy such as Dunnigan-type familial partial lipodytsrophy (FPLD) and HutchinsonGilford progeria syndrome (HGPS), respectively [reviewed in (Broers et al. 2006; Verstraeten et al. 2007)]. In contrast to FPLD, HGPS is mostly caused by a de novo $1824 \mathrm{C}>\mathrm{T}$ 

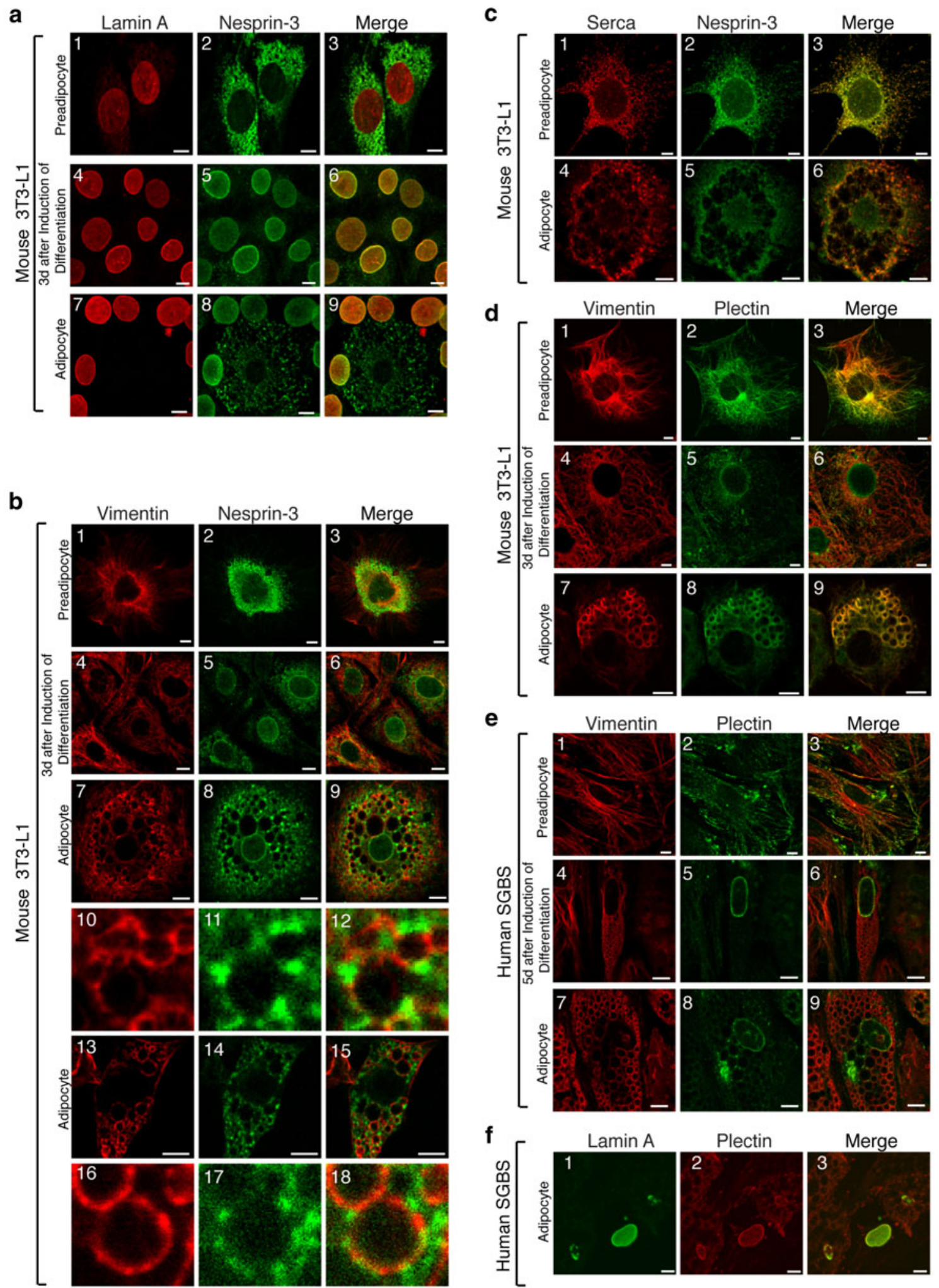

e
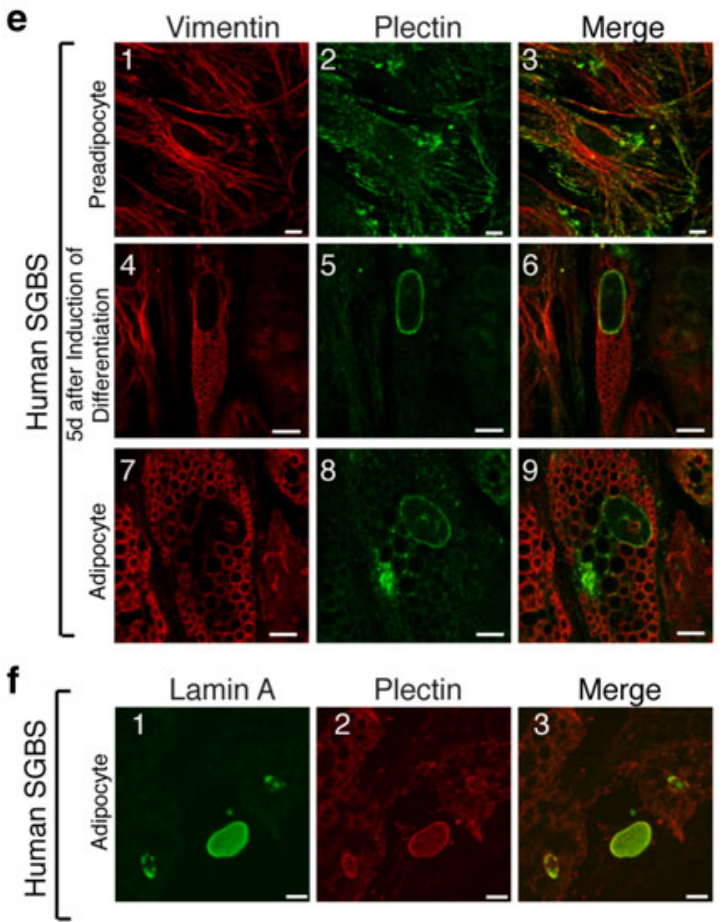

mutation in $L M N A$ that results in a permanently farnesylated protein called progerin, which affects the polymerization of the nuclear lamina resulting in stiffer nuclei (Dahl

et al. 2006; Verstraeten et al. 2008). Breakdown of the nuclear lamina is an early event in adipogenesis and henceforward, may be a prerequisite for adipose 
4 Fig. 3 Reorganization of the nuclear-cytoskeleton junction during adipogenesis. Preadipocytes express lamin A at the nuclear rim (a1) and nesprin-3 in the ER (a2, merge a3). After induction to fat cell differentiation, the expression of lamin $\mathrm{A}$ is enhanced at the nuclear rim (a4) and nesprin-3 redistributes towards the nuclear rim (a5), where both co-localize (merge in a6). Due to fragmentation of the nuclear lamina network (Fig. 2), a large part of the mature adipocytes totally lack lamin A (a7) and nesprin-3 (a8) at the nuclear rim (merge in a9). Vimentin (b1, $4,7,10,13,16)$ and nesprin-3 $(\mathbf{b} 2,5,8,11,14,17)$ do not co-localize throughout adipose conversion (merge in b3, 6, 9, 12, 15, 18), however, they do form an intricate network surrounding lipid droplets (b12, detail of b9; b18, detail of b15). Double-labeling studies of $\mathrm{ER} \mathrm{Ca}^{2+}$-ATPase SERCA2 $(\mathbf{c 1}, \mathbf{4})$ with nesprin-3 $(\mathbf{c 2}, \mathbf{5})$ confirms the organization of nesprin-3 in the ER of preadipocytes and adipocytes (merge in $\mathbf{c 3 , 6}$ ). Plectin (d2, e2) partially co-localizes with vimentin (d1, e1) in preadipocytes (merge in $\mathbf{d 3}, \mathbf{e 3}$ ). Upon differentiation, plectin $(\mathbf{d 5}, \mathbf{e 5})$ reorganizes to the nuclear rim, where little to no co-localization with vimentin occurs $(\mathbf{d 4}, \mathbf{e 4}$; merge in $\mathbf{~ d 6 , ~ e 6 ) . ~ I n ~ a d i p o c y t e s , ~ p l e c t i n ~}(\mathbf{d 8}, \mathbf{e 8})$ co-localizes (partially) with vimentin (d7, e7) around fat droplets (merge in d9, e9). Instead of its total loss, fragmentation of the lamin A network can remain in adipocytes (f1), where it partially co-localizes with plectin (f2, merge in f3). Bar $10 \mu \mathrm{m}$. Single confocal sections are presented for a7-9, b1-3, b7-18, c1-3, e4-9. The other images result from $Z$-projection of the representative confocal image stack conversion. It is conceivable that progerin impairs the essential reorganization of the nuclear lamina network and thereby, abolishes fat cell differentiation causing generalized lipodystrophy in HGPS. This hypothesis is supported by the fact that human mesenchymal stem cells expressing progerin showed markedly reduced differentiation along the adipogenic lineage, with lipid accumulation only in those hMSCs expressing very low levels of progerin (Scaffidi and Misteli 2008).

In conclusion, our findings show a dramatic reorganization of the nuclear lamina and its coupling to the vimentin intermediate filament network during adipose conversion. We suggest that adipogenesis may therefore serve as a model system in which to examine the LINC complex. This dynamic reorganization of the nuclear lamina network in the process of fat cell differentiation is an important insight that may provide new venues for research in and treatment of $L M N A$-associated lipodystrophy and other adipose tissue diseases such as obesity.
Fig. 4 Reorganization of the cytoskeleton during adipogenesis. Actin stress fibers (a1, b1) reorganize into a cortical network lining membrane caves (a2, arrow; insert for detail) projecting into the perinuclear area upon induction of differentiation. Adipocytes show fewer perinuclear caves $(\mathbf{a 3}, \mathbf{b 2})$. Vimentin $(\mathbf{a} 4, \mathbf{b 3})$ reorganizes to cage-like structures surrounding lipid droplets $(\mathbf{a 5}, \mathbf{b 4})$ or a cortical network in adipocytes (a6). Microtubules $(\mathbf{a} 7, \mathbf{b 5})$ reorganize into a network under the plasma membrane and in between lipid droplets (a8, a9, b6). The MTOC remains closely positioned to the nucleus (a10-11, b7-8). Nuclei are counterstained with PI (red). Bar $10 \mu \mathrm{m}$. Single confocal sections are presented for a2, 4, 5, 8, 9 and b4, 6. The other images result from $Z$-projection of the representative confocal image stack a
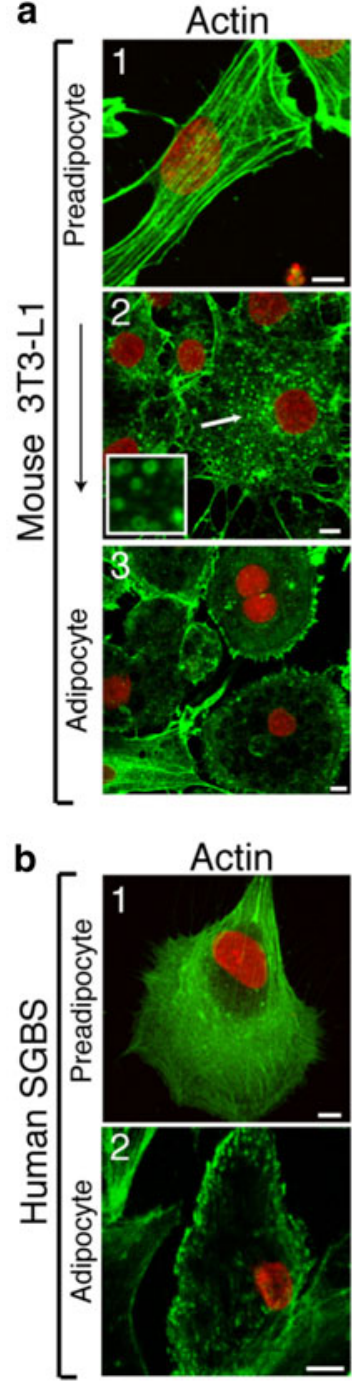
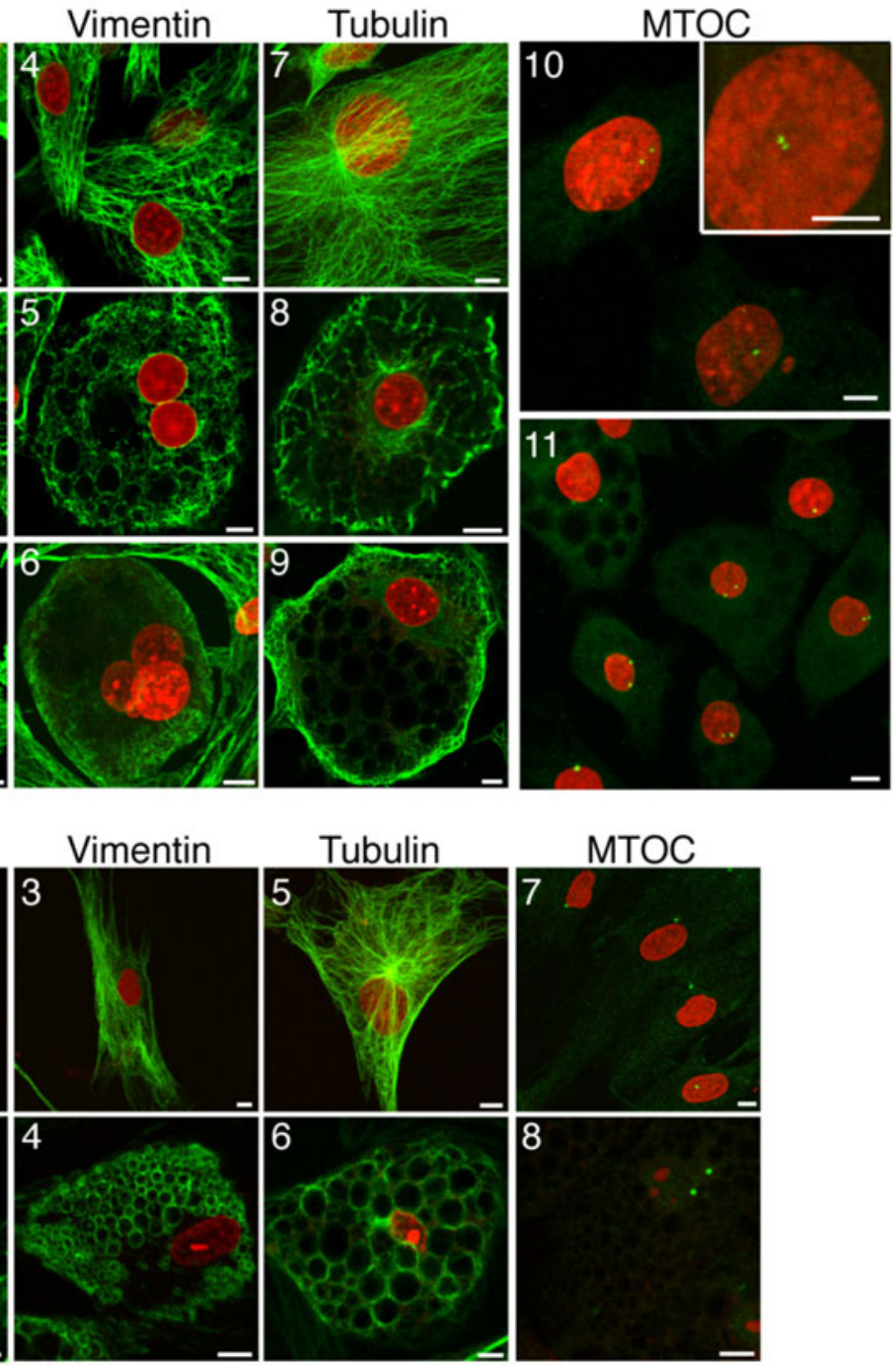

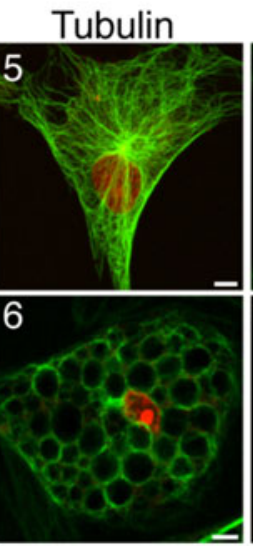

MTOC

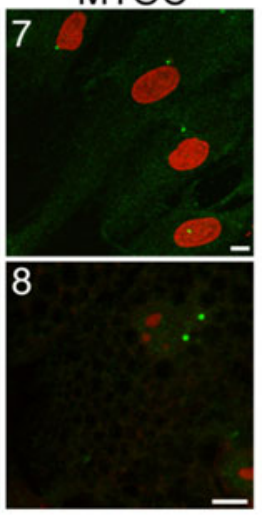



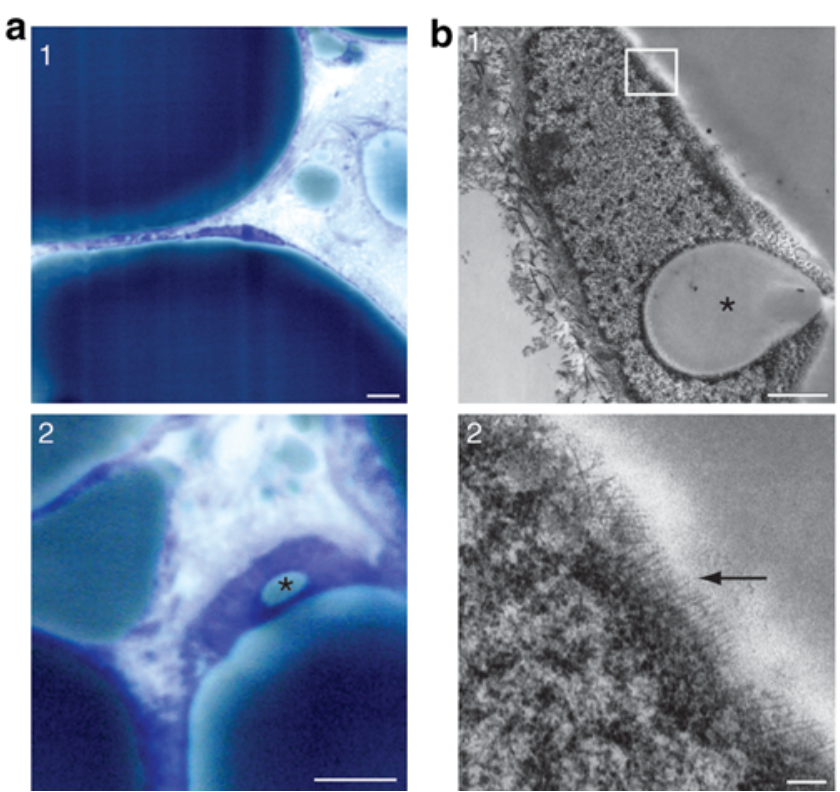

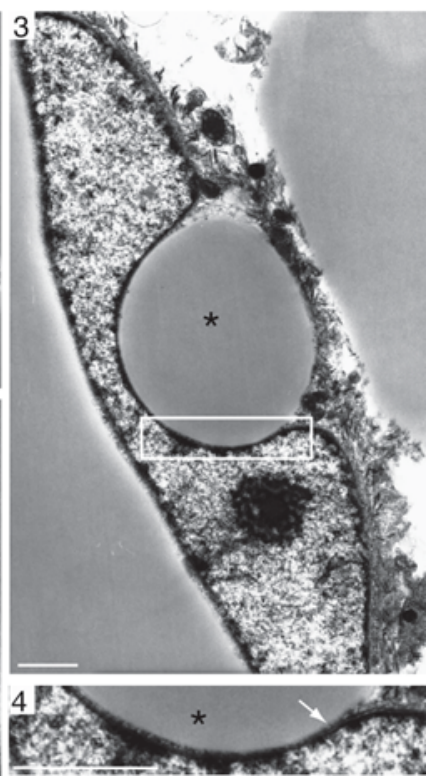

Fig. 5 Structural analysis of fat cells in a human adipose tissue specimen. Light $(\mathbf{a 1}, \mathbf{2})$ and electron (b1-4) microscopy shows thin and peripherally located nuclei (a1), indented by lipid droplets (asterisk in a2, b1, 3-4). The lipid bi-layer of the nuclear envelope in $\mathbf{b 3}$ (detail of white box in b3 provided in b4) confirms the nuclear indentation. In between the adipocyte lipid mass and the peripherally

Acknowledgments This work is supported by a research grant of the University Hospital Maastricht to VV. MvS is financially supported by Barrier Therapeutics and a research grant of the University Hospital Maastricht. The authors wish to acknowledge Arnoud Sonnenberg (Amsterdam) for kindly providing the antibody to nesprin-3, Katsushi Owaribe (Nagoya) and W.H. Irwin McLean (Dundee) for kindly providing the HD-121 antibody, Jean-Claude Courvalin (Paris) for kindly providing the antibody to lamin B1, Christoph Viebahn (Bonn) for kindly providing antibody BV-1118, Georg Krohne (Würzburg) for kindly providing antibody X223, Chris Hutchinson (Durham) for kindly providing antibody Jol-2. The authors wish to thank Jan Lammerding (Boston, USA) and Maria Lucia Lombardi (Boston, USA) for helpful discussions. The Netherlands Organization for Scientific Research (NWO, project 901-28-134) is acknowledged for financial support for microscopic equipment and imaging software.

Open Access This article is distributed under the terms of the Creative Commons Attribution Noncommercial License which permits any noncommercial use, distribution, and reproduction in any medium, provided the original author(s) and source are credited.

\section{References}

Akter R, Rivas D, Geneau G, Drissi H, Duque G (2009) Effect of lamin A/C knockdown on osteoblast differentiation and function. J Bone Miner Res 24:283-293

Boguslavsky RL, Stewart CL, Worman HJ (2006) Nuclear lamin A inhibits adipocyte differentiation: implications for Dunnigantype familial partial lipodystrophy. Hum Mol Genet 15:653-663

Bouwman F, Renes J, Mariman E (2004) A combination of protein profiling and isotopomer analysis using matrix-assisted laser located nucleus a filamentous network can be seen (detail of white box in b1 provided in b2; arrow points to filamentous structures). Interestingly, no such filamentous network surrounds the small lipid droplets that indent the nucleus (b4). Scale bar in a1, 2: $5 \mu \mathrm{m}$, in b1, 3, 4: $1 \mu \mathrm{m}$ and in b2: $100 \mathrm{~nm}$

desorption/ionization-time of flight mass spectrometry reveals an active metabolism of the extracellular matrix of 3T3-L1 adipocytes. Proteomics 4:3855-3863

Broers JL, Ramaekers FC, Bonne G, Yaou RB, Hutchison CJ (2006) Nuclear lamins: laminopathies and their role in premature ageing. Physiol Rev 86:967-1008

Capanni C, Mattioli E, Columbaro M, Lucarelli E, Parnaik VK, Novelli G, Wehnert M, Cenni V, Maraldi NM, Squarzoni S, Lattanzi G (2005) Altered pre-lamin A processing is a common mechanism leading to lipodystrophy. Hum Mol Genet 14:1489-1502

Carmo-Fonseca M, Kern H, Hurt EC (1991) Human nucleoporin p62 and the essential yeast nuclear pore protein NSP1 show sequence homology and a similar domain organization. Eur J Cell Biol 55:17-30

Crisp M, Liu Q, Roux K, Rattner JB, Shanahan C, Burke B, Stahl PD, Hodzic D (2006) Coupling of the nucleus and cytoplasm: role of the LINC complex. J Cell Biol 172:41-53

Dahl KN, Scaffidi P, Islam MF, Yodh AG, Wilson KL, Misteli T (2006) Distinct structural and mechanical properties of the nuclear lamina in Hutchinson-Gilford progeria syndrome. Proc Natl Acad Sci USA 103:10271-10276

Fischer-Posovszky P, Newell FS, Wabitsch M, Tornqvist HE (2008) Human SGBS cells-a unique tool for studies of human fat cell biology. Obes Facts 1:184-189

Franke WW, Hergt M, Grund C (1987) Rearrangement of the vimentin cytoskeleton during adipose conversion: formation of an intermediate filament cage around lipid globules. Cell 49:131141

Hieda Y, Nishizawa Y, Uematsu J, Owaribe K (1992) Identification of a new hemidesmosomal protein, HD1: a major, high molecular mass component of isolated hemidesmosomes. J Cell Biol 116:1497-1506

Hoger TH, Zatloukal K, Waizenegger I, Krohne G (1990) Characterization of a second highly conserved B-type lamin present in 
cells previously thought to contain only a single B-type lamin. Chromosoma 99:379-390

Lammerding J, Fong LG, Ji JY, Reue K, Stewart CL, Young SG, Lee RT (2006) Lamins A and C but not lamin B1 regulate nuclear mechanics. J Biol Chem 281:25768-25780

Markiewicz E, Tilgner K, Barker N, van de Wetering M, Clevers H, Dorobek M, Hausmanowa-Petrusewicz I, Ramaekers FC, Broers JL, Blankesteijn WM, Salpingidou G, Wilson RG, Ellis JA, Hutchison CJ (2006) The inner nuclear membrane protein emerin regulates beta-catenin activity by restricting its accumulation in the nucleus. EMBO J 25:3275-3285

Parton RG, Molero JC, Floetenmeyer M, Green KM, James DE (2002) Characterization of a distinct plasma membrane macrodomain in differentiated adipocytes. J Biol Chem 277:46769-46778

Renes J, Bouwman F, Noben JP, Evelo C, Robben J, Mariman E (2005) Protein profiling of 3T3-L1 adipocyte differentiation and (tumor necrosis factor alpha-mediated) starvation. Cell Mol Life Sci 62:492-503

Rosen ED, MacDougald OA (2006) Adipocyte differentiation from the inside out. Nat Rev Mol Cell Biol 7:885-896

Scaffidi P, Misteli T (2008) Lamin A-dependent misregulation of adult stem cells associated with accelerated ageing. Nat Cell Biol 10:452-459

Tilgner K, Wojciechowicz K, Jahoda C, Hutchison C, Markiewicz E (2009) Dynamic complexes of A-type lamins and emerin influence adipogenic capacity of the cell via nucleocytoplasmic distribution of beta-catenin. J Cell Sci 122:401-413

Verstraeten VL, Broers JL, van Steensel MA, Zinn-Justin S, Ramaekers FC, Steijlen PM, Kamps M, Kuijpers HJ, Merckx D, Smeets HJ, Hennekam RC, Marcelis CL, van den Wijngaard A (2006) Compound heterozygosity for mutations in LMNA causes a progeria syndrome without prelamin A accumulation. Hum Mol Genet 15:2509-2522

Verstraeten VL, Broers JL, Ramaekers FC, van Steensel MA (2007) The nuclear envelope, a key structure in cellular integrity and gene expression. Curr Med Chem 14:1231-1248

Verstraeten VL, Ji JY, Cummings KS, Lee RT, Lammerding J (2008) Increased mechanosensitivity and nuclear stiffness in Hutchinson-Gilford progeria cells: effects of farnesyl transferase inhibitors. Aging Cell 7:383-393

Wabitsch M, Brenner RE, Melzner I, Braun M, Moller P, Heinze E, Debatin KM, Hauner H (2001) Characterization of a human preadipocyte cell strain with high capacity for adipose differentiation. Int J Obes Relat Metab Disord 25:8-15

Wilhelmsen K, Litjens SH, Kuikman I, Tshimbalanga N, Janssen H, van den Bout I, Raymond K, Sonnenberg A (2005) Nesprin-3, a novel outer nuclear membrane protein, associates with the cytoskeletal linker protein plectin. J Cell Biol 171:799-810 